since no official scoring system such as the Behcet's Disease Current Activity Form (BDCAF) was available at the start of anti-TNF $\alpha$ treatment in our centre.

It is unknown how long anti-TNF $\alpha$ treatment must be given, but anti-TNF $\alpha$ treatment in patients with rheumatic arthritis is continued for $>2$ years and continued until there is a settled response. ${ }^{10}$ In our patients, infliximab was discontinued after complete response of $>3$ months or acceptable improvement of (eye) symptoms. In five of the six patients, relapses after infliximab did not necessitate immediate restart of anti-TNF $\alpha$ treatment. In this period (mean duration 562, range 1361093 days), immunosuppressive therapy could be adjusted until the symptoms required a restart of anti-TNF $\alpha$ treatment. Adalimumab was considered to be equal potential, but more convenient, and was added in cases of severe relapse with patients' informed consent. In addition, formation of autoantibodies to infliximab when restarted was considered. All patients responded and most of them showed dramatic and quick improvement. Subsequently, immunosuppressive therapy could again be tapered (table 1 ).

Patient 6 had a severe BD-associated colitis and was periodically treated with infliximab and other immunosuppressive agents for nearly 3 years. Despite intensified immunosuppressive therapy, the colitis worsened and became refractory and life threatening. Subsequently, a high dose of adalimumab $40 \mathrm{mg} /$ week was started subcutaneously, yielding a complete response of $>1$ year. Adalimumab was briefly combined with $30 \mathrm{mg}$ of prednisone, which was tapered rapidly to prevent central retinal serosa ablation that developed in a previous period in which steroids had been used. Later, mesalazine and rectal budenoside were also given. Apart from some minor flares, the patient remained stable for nearly 2 years. Until now, all patients are receiving adalimumab, except patient 5 who discontinued 4 months after complete remission was achieved (table 1). In general, few side effects were observed. Three patients (1,3 and 6) developed lichenoidlike lesions that were treated with local steroids by a dermatologist.

This report on patients with treatment refractory BD indicates that adalimumab treatment is promising and can be prescribed safely for a prolonged period. To our knowledge, this is the first case series in which patients with BD with systemic disease treated with adalimumab are presented. More studies on this subject are warranted.

\section{Authors' affiliations}

J A M van Laar, P L A van Daele, A Jamnitski, P M van Hagen,

T Missotten, G S Baarsma, Erasmus University Medical Center, Rotterdam, The Netherlands

Competing interests: PMvH has cooperated in a European study on patients with uveitis treated with infliximab that was sponsored by Centocor. JAMvL and $\mathrm{PMvH}$ were in part sponsored to visit the 12th international Behçet's congress in Lisbon by Abbott BV where JAMvL presented these data to the international investigators on Behçet's disease.

Correspondence to: Dr J A M van Laar, Room D-419, Erasmus University Medical Center, Gravendijkwal 230, Rotterdam 3015 CE, The Netherlands; j.vanlaar@erasmusmc.nl

Accepted 2 November 2006

Published Online 23 November 2006

\section{REFERENCES}

1 Verians GM, van Hagen PM, van der Kooi A, Osterhaus AD, Baarsma GS. Vgamma9Vdelta2 $\mathrm{T}$ cells recovered from eyes of patients with Behcet's disease recognize non-peptide prenyl pyrophosphate antigens. J Neuroimmunol 2002;130:46-54

2 Evereklioglu C. Current concepts in the etiology and treatment of behcet disease Surv Ophthalmol 2005:50:297-350.

3 Atzeni F, Sarzi-Puttini P, Doria A, laccarino L, Capsoni F. Potential off-label use of infliximab in autoimmune and non-autoimmune diseases: a review. Autoimmun Rev 2005;4:144-52.

4 Sakane T, Takeno M, Suzuki N, Inaba G. Behcet's disease. N Engl J Med 1999:341:1284-91.

5 Stokes DG, Kremer JM. Potential of tumor necrosis factor neutralization strategies in rheumatologic disorders other than rheumatoid arthritis. Semin Arthritis Rheum 2003;33:1-18

6 Lindstedt EW, Baarsma GS, Kuijpers RW, van Hagen PM. Anti-TNF-alpha therapy for sight threatening uveitis. Br J Ophthalmol 2005;89:533-6.

7 Melikoglu M, Fresko I, Mat C, Ozyazgan Y, Gogus F, Yurdakul S, et al. Shortterm trial of etanercept in Behcet's disease: a double blind, placebo controlled study. J Rheumatol 2005;32:98-105.

8 Mushtaq B, Saeed T, Situnayake RD, Murray PI. Adalimumab for sightthreatening uveitis in Behcet's disease. Ey. Published Online First: 7 April 2006 doi:10.1038/si.eye.6702352.

9 Van Laar JAM, Jamnitski A, Baarsma G, van Daele PLA, van Hagen PM. Behçet's disease and the possibilities of modern tumour necrosis factor inhibiting medication. Ned Tiidschr Geneeskd 2006;150:705-9.

10 Hochberg MC, Lebwohl MG, Plevy SE, Hobbs KF, Yocum DE. The benefit/risk profile of TNF-blocking agents: findings of a consensus panel. Semin Arthritis Rheum 2005;34:819-36.

\title{
Autosplenectomy: rare syndrome in autoimmunopathy
} J Leipe, A J Hueber, S Kallert, J Rech, H Schulze-Koops

A utosplenectomy has been described in association with systemic lupus erythematosus (SLE). ${ }^{1}$ Although in patients with SLE small or atrophic spleens are usually seen, the complete absence of the spleen has been observed rarely. Here, we report a patient with an 18-year history of autoimmunopathy who developed anatomical asplenia during the disease.

A 51-year-old Caucasian woman was first diagnosed in 1988 as having autoimmunopathy of incompletely defined aetiology with antinuclear antibodies (ANA) positivity and immunocomplexes in conjunction with reduced complement levels and serositis. The patient presented with massive pleural and pericardial effusions.
Laboratory studies showed normal blood counts. Antibodies to extractable nuclear antigens or to phospholipids (lupus anticoagulant and anti-cardiolipin antibodies) could not be detected. Although suggestive of having SLE, the patient did not fulfill the classification criteria for the disease at any given time. ${ }^{2}$ Notably, the spleen was normal in all respects as confirmed by ultrasound and CT scan, at the time of initial contact. Treatment was initiated with glucocorticoids and plasmapheresis, with good clinical response. Because of unresponsiveness to or unbearable toxicity

Abbreviations: $\mathrm{AlH}$, autoimmune hepatitis; $\mathrm{ANA}$, antinuclear antibody; SLE, systemic lupus erythematosus 

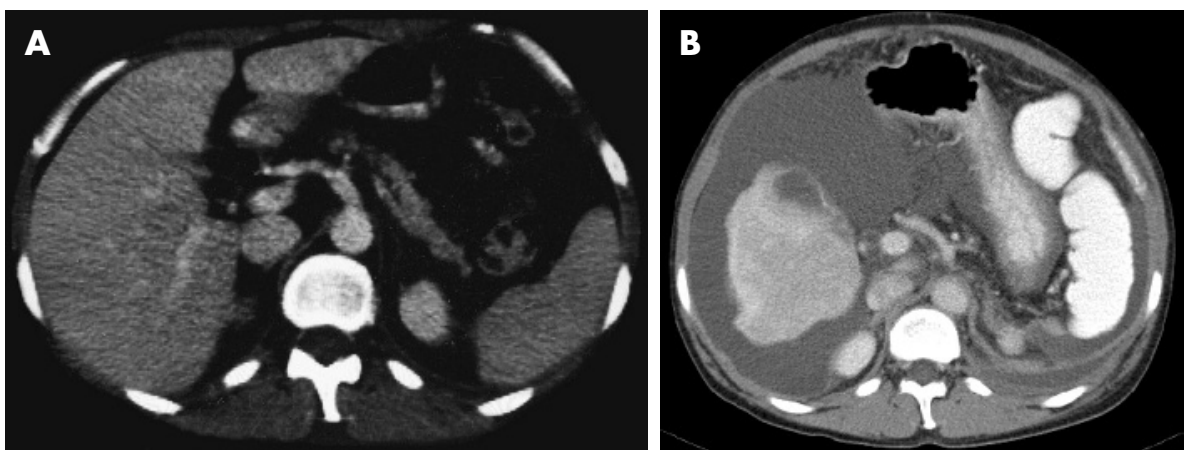

Figure 1 Demonstration of the presence and absence of the spleen. (A) Abdominal CT scan performed in 1992 showing a morphologically normal spleen. (B) Fourteen years later, the spleen is diminished; of note is the appearance of ascites and an atrophic nodular liver. of subsequently started treatment with azathioprine, cyclophosphamide and ciclosporin, a regimen of repeated plasmapheresis during the following years was used to control pleural and pericardial effusions efficiently.

The patient was admitted to the hospital 18 years later because of the development of ascites during the preceding 2 weeks. Laboratory studies revealed an increased $C$ reactive protein serum level $(3,2 \mathrm{mg} / \mathrm{dl})$, increased levels of immune complexes (IgG, IgM, IgA), decreased serum complement C3 and $\mathrm{C} 4$ and raised liver function tests. Peripheral blood counts were within normal limits. Howell-Jolly bodies were noticed in the blood smear. Indirect immunofluorescence showed a significant titre of circulating ANA (1:100) whereas antimitochondrial antibody, smooth muscle actin antibody and liver, kidney microsomal-1-antibodies were absent. As in the previous tests, extra nuclear antigen and anti-phospholipid antibodies were undetectable.

Ultrasound, CT scan and MRI demonstrated ascites, pleural effusions, pericardial effusion and an atrophic nodular-appearing liver with inhomogeneous contrast enhancement. Surprisingly, in contrast with previous investigations, the spleen was undetectable by ultrasound, CT scan and MRI (fig l).

Liver biopsy revealed the presence of lymphocytic infiltrations, collapse fibrosis and central portal bridging necrosis, a picture potentially consistent with autoimmune hepatitis (AIH). After excluding other conditions predisposing to hepatitis, the patient fulfilled the criteria for AIH as defined by the International Autoimmune Hepatitis Group. ${ }^{3}$

Treatment for AIH with $1 \mathrm{mg} / \mathrm{kg}$ prednisolone with subsequent tapering was initiated, which yielded a partial clinical response.

Whereas functional asplenia occurs in about $5 \%$ of patients with SLE, anatomical asplenia has been seen in only a few cases. ${ }^{4}$ The mechanism by which autosplenectomy develops in autoimmune diseases is rather unclear, but may relate to vasculitis with increased levels of immune complexes and subsequent silent splenic infarctions. ${ }^{4}$ Our patient had increased levels of immune complexes since the first clinical appearance of the autoimmune disease. Although there were no obvious signs of vasculitis, it is tempting to interpret the decrease in complement and presence of ANA and immune complexes as findings suggestive of unrecognised vasculitis that may have caused microinfarction and, hence, incremental atrophy of the spleen. Alternatively, AIH may have caused the anatomical asplenia. Notably, there is only one report demonstrating functional asplenia in a patient with non-autoimmune, aggressive hepatitis. ${ }^{5}$

In summary, we present the first case of a patient with anatomical autosplenectomy in the setting of an autoimmunopathy different from SLE.

\section{Authors' affiliations}

A J Hueber, S Kallert, J Rech, Department of Internal Medicine III, University of Erlangen-Nuremberg, Erlangen, Germany

J Leipe, H Schulze-Koops, Medizinische Poliklinik-Rheumatologie, University of Munich, Germany

Competing interests: None declared.

Correspondence to: Dr H Schulze-Koops, Medizinische Poliklinik Rheumatologie, University of Munich, Pettenkoferstraße 8a, 80336 Munich, Germany;

hendrik.schulze-koops@med.uni-muenchen.de

Accepted 2 November 2006

\section{REFERENCES}

1 Santilli D, Govoni M, Prandini N, Rizzo N, Trotta F. Autosplenectomy and antiphospholipid antibodies in systemic lupus erythematosus: a pathogenetic relationship? Semin Arthritis Rheum 2003;33:125-33.

2 Tan EM, Cohen AS, Fries JF, Masi AT, McShane DJ, Rothfield NF, et al. The 1982 revised criteria for the classification of systemic lupus erythematosus. Arthritis Rheum 1982;25:1271-7.

3 Alvarez F, Berg PA, Bianchi FB, Bianchi L, Burroughs AK, Cancado EL, et al. International Autoimmune Hepatitis Group Report: review of criteria for diagnosis of autoimmune hepatitis. J Hepatol 1999;31:929-38.

4 Dillon AM, Stein HB, English RA. Splenic atrophy in systemic lupus erythematosus. Ann Intern Med 1982;96:40-3.

5 Dhawan VM, Spencer RP, Sziklas JJ. Reversible functional asplenia in chronic aggressive hepatitis. J Nucl Med 1979;20:34-6. 